\title{
Micromagnetics of submicron (110) Fe elements
}

\author{
Luc Thomas and Stuart S. P. Parkin \\ IBM Research Division, Almaden Research Center, 650 Harry Road, San Jose, California 95120 \\ Jun Yu, Ulrich Rüdiger, and Andrew D. Kent \\ Department of Physics, New York University, New York, New York 10003
}

(Received 4 August 1999; accepted for publication 1 December 1999)

\begin{abstract}
The magnetization processes and the domain structure of epitaxial (110) Fe submicron-sized elements are studied experimentally and with micromagnetic simulations. Magnetic force microscopy and Kerr magnetometry results are in good agreement with the simulations, thus allowing the development of a consistent picture of the micromagnetics of these systems.
\end{abstract}

With the recent development of magneto-electronic devices such as magnetic random access memory ${ }^{1}$ and giant magnetoresistive heads, ${ }^{2}$ it has become crucial to understand in detail the domain structure and magnetization reversal of lithographically defined micron-sized elements. ${ }^{3-6}$ Here we report results on epitaxial Fe elements. Good agreement between experimental results and micromagnetic calculations allows a precise description of the magnetization processes in these systems.

(110) oriented 50-nm-thick Fe films were prepared on $a$-axis oriented sapphire substrates using ultrahigh vacuum electron-beam evaporation techniques as described elsewhere. ${ }^{7}$ Magnetic measurements of the unpatterned films ${ }^{8}$ indicate two significant contributions to the magnetic anisotropy, namely, (1) a cubic term $K_{1}=6.3 \times 10^{5} \mathrm{erg} / \mathrm{cm}^{3}$ and (2) an uniaxial term $K_{u}=3.0 \times 10^{5} \mathrm{erg} / \mathrm{cm}^{3}$, both with the magnetic easy axis along [001]. The Fe films were patterned into arrays of elements of various sizes and shapes using electron beam lithography and ion milling. ${ }^{8,9}$ In this letter we focus on rectangular particles each $0.5 \mu \mathrm{m}$ width and $1.5 \mu \mathrm{m}$ long. The elements are separated from one another by four times their width and three times their length so that the dipolar interactions between neighboring particles are small. Indeed, we estimate the dipolar fields to be less than 10 Oe from micromagnetic calculations, assuming the particles are saturated along their long axes.

Magnetization processes were investigated experimentally using a magneto-optical Kerr effect (MOKE) magnetometer and magnetic force microscopy (MFM). CoCr MFM tips were used which were magnetized normal to the surface of the films in order to sense the out-of-plane component of the magnetic stray field. Note that MOKE was performed on arrays of elements while the MFM images were obtained on single particles.

Micromagnetic calculations were performed on Fe particles of the same size and shape as those investigated experimentally using the LLG Micromagnetics Simulator ${ }^{\mathrm{TM}}$ code developed by M. R. Scheinfein. ${ }^{10}$ The magnetization distribution is approximated by a cubic mesh with a cell size of $(10 \mathrm{~nm})^{3}$ so that the simulation of one particle involves $150 \times 50 \times 5$ cells. In some cases cells were removed at each of the four corners to mimic shape imperfections found experimentally, but the rectangular symmetry was preserved. The equilibrium magnetization is found by solving the dynamic Landau-Lifshitz-Gilbert (LLG) equation. The effec- tive field acting on each cell is obtained from the derivative of the free energy of the system with regard to the local magnetization, where the free energy includes Zeeman, magnetostatic, exchange, and magnetocrystalline energy terms. Values of saturation magnetization $M_{s}=1714 \mathrm{emu} / \mathrm{cm}^{3}$, and exchange stiffness $A=2.110^{-6} \mathrm{erg} / \mathrm{cm}$ of bulk iron were used in the simulations. As we are interested in quasistatic domain patterns, the dynamic LLG equation was solved assuming a strong damping constant $\alpha=1$. Special attention was devoted to the influence of the cell size and the convergence criteria on the results. In particular, tests performed using finer meshes gave similar results. The convergence criterion used was based on the largest allowed angular variation of the magnetization of a single cell during two consecutive iterations. A value of $10^{-6}$ was found to be satisfactory.

The competition between the magnetocrystalline anisotropy (whose easy axis is, by design, perpendicular to the long axis of the particle) and the shape anisotropy energies leads to complex domain structures at low fields. MFM images obtained in zero field after saturation both along and perpendicular to the particle long axis (see insets in Fig. 1) show flux-closed multidomain patterns. Interestingly, even though these two remanent states are qualitatively similar, they do not exhibit the same number of vortices. After longitudinal saturation, there are three vortices while there are only two after transverse saturation. Note that these two patterns are representative of most of the particles in the array. However, different domain structures are observed for some of the particles perhaps due to irregularities in particle shape. ${ }^{8}$ MOKE hysteresis loops measured on the array along and perpendicular to the particle long axis (see Fig. 1) also exhibit the characteristic features of flux-closed domain structures. The magnetization $M$ drops rapidly when vortices are nucleated at the nucleation field $H_{n}$ (see Fig. 1) and continues to evolve until the vortices are expelled at the annihilation field $H_{a}$. Both $H_{n}$ and $H_{a}$ depend on the orientation of the applied field. Along the particle long axis, $H_{n}$ $\sim 200 \mathrm{Oe}$ and $H_{a} \sim 400 \mathrm{Oe}$, whereas along the perpendicular direction, $H_{n} \sim 350 \mathrm{Oe}$ and $H_{a} \sim 1300 \mathrm{Oe}$. Note that these values are not well defined, since the features of the hysteresis loops are broadened by likely variations in properties of the individual particles. The high field $M$ vs $H$ slope measured on both hysteresis loops (Fig. 1) suggests that the magnetization does not saturate after the vortices have been expelled from the structure. (Note that these high field slopes 

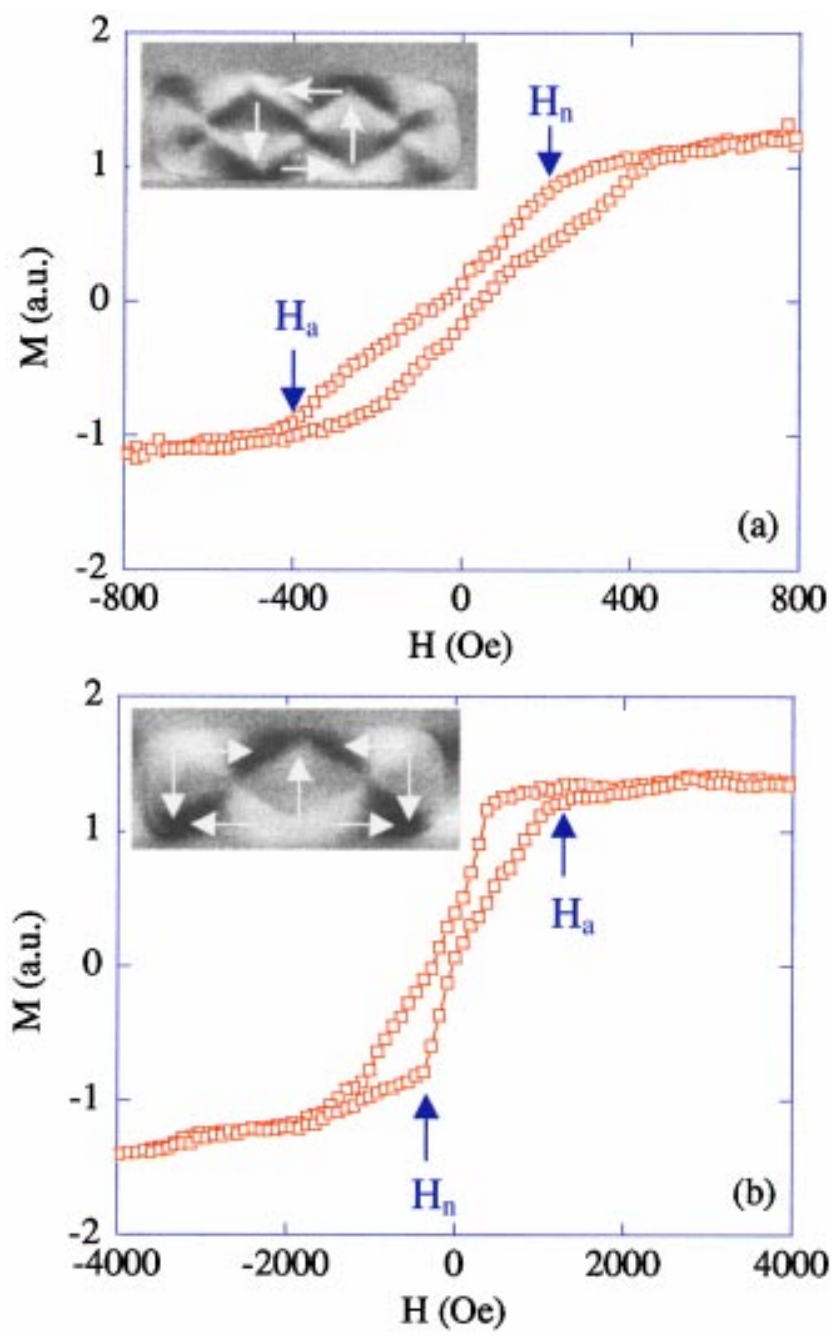

FIG. 1. (Color) Hysteresis loops obtained by Kerr magnetometry with a magnetic field applied (a) along and (b) perpendicular to the long axis of the particles. The insets show the zero-field domain structure imaged by MFM corresponding to these field orientations.

may be affected by experimental MOKE artifacts). The theoretical saturation fields can be calculated from the demagnetization factors for the prismatic shape of the $\mathrm{Fe}$ elements. ${ }^{11}$ For the aspect ratio, $x=30 \times y=10 \times z=1$, the calculated demagnetization factors are, respectively, $N_{x}$ $=0.0357, N_{y}=0.1117$ and $N_{z}=0.8526$. The saturation field $H_{s}$ is the sum of the demagnetizing and anisotropy fields along the corresponding direction. Values of $H_{s}$, perpendicular and parallel to the particle's long axis, are estimated to be $\sim 2.4$ and $\sim 1.3 \mathrm{kOe}$, respectively, much larger that the corresponding annihilation fields $H_{a}(\sim 1.3$ and $0.4 \mathrm{kOe}$, respectively).

We have calculated the hysteresis loops for both longitudinal and transverse field orientations, starting with a large positive field along either of these directions. The initial state was chosen to be uniformly magnetized along the field direction. The quasi-equilibrium magnetization was then calculated for a series of fields within the hysteresis loop.

Detailed results are shown in Figs. 2 and 3 for the transverse and longitudinal cases, respectively. The domain patterns at various fields are shown as insets. For the transverse case, there are four characteristic regimes. First, for large positive fields $(H>1 \mathrm{kOe})$, the magnetization is mainly parallel to the field and lies perpendicular to the long axis of the

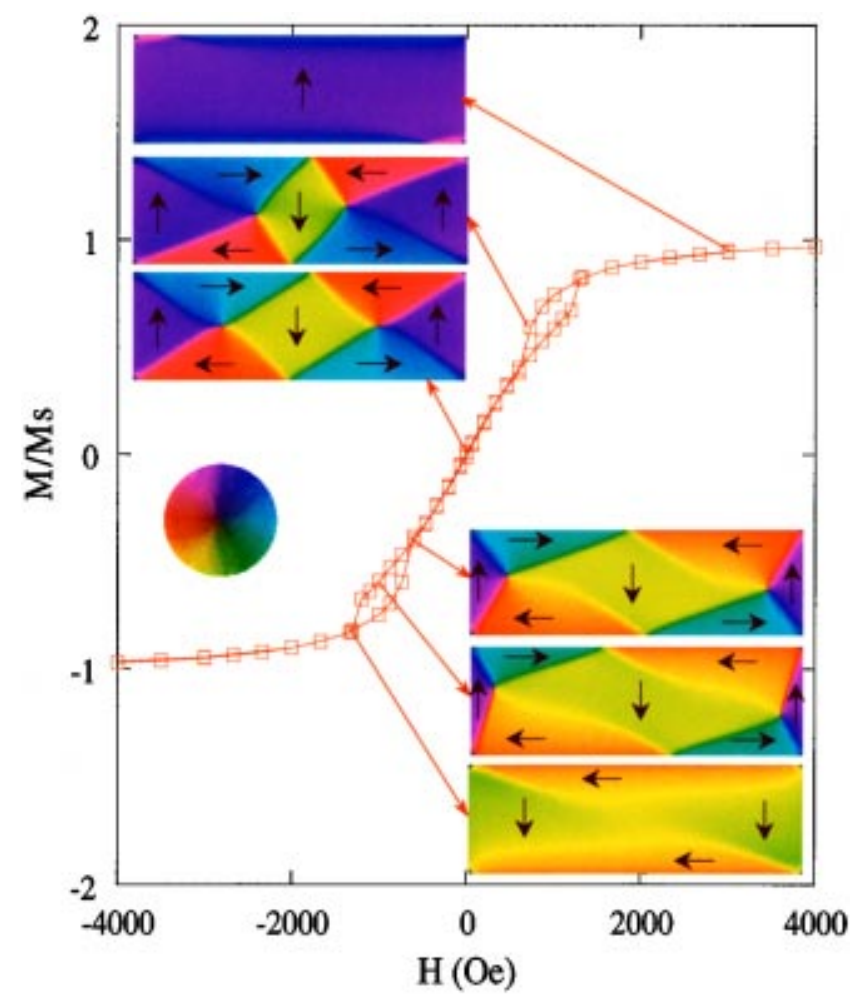

FIG. 2. (Color) Simulated hysteresis loop for field applied transverse to the particle long axis. In the inset are shown domain structures for several representative values of the applied field. Black arrows give a rough indication of the magnetization direction. The color wheel gives a more precise description of the magnetization orientation. The four in-plane directions are red (right), yellow (down), green (left), and blue (up).

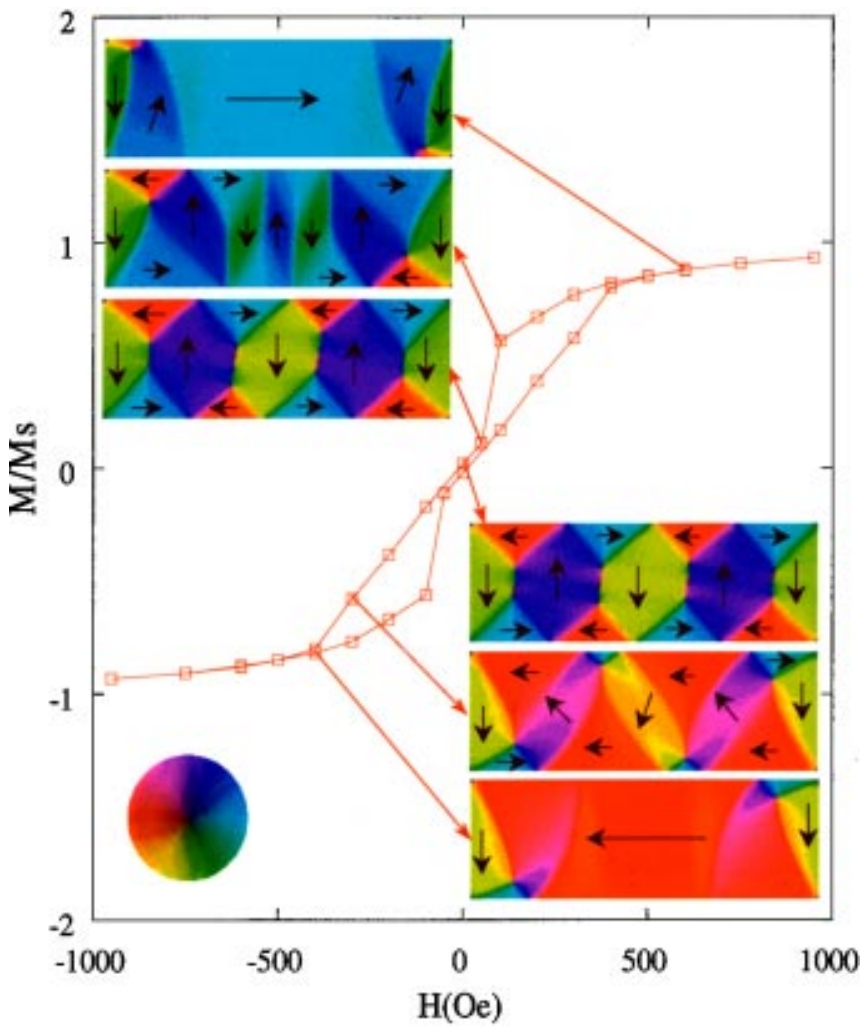

FIG. 3. (Color) Simulated hysteresis loop for field applied along the particle long axis. In the inset are shown the domain structure for several values of the applied field. Color codes are the same as in Fig. 2. 
particles. However, this is not a single domain state. Along the two long sides of particle, the magnetization is tilted towards the edges to reduce the magnetostatic energy. Along the two short sides, the magnetization is parallel to the surface, so that all the Zeeman, magnetostatic and magnetocrystalline energies are minimized. Thus, since these two edge domains are very stable, when the field is decreased to $H$ $\sim 750 \mathrm{Oe}$, a reversed domain is nucleated from the middle of the particle. This process gives rise to a flux-closed structure which contains two vortices. As the field is reduced further through zero field, the reversed domain progressively expands towards the short edges of the particles. This process is reversible, and its susceptibility is small since the gain in Zeeman and magnetocrystalline energy is compensated by the higher cost in magnetostatic energy. When the field reaches $H \sim-1200 \mathrm{Oe}$, the two vortices are expelled from the particle which attains near saturation in the negative direction.

For the longitudinal case, the magnetization reversal process is qualitatively similar. As the field applied along the long axis is reduced from saturation, the magnetization along the two long sides of the particle is parallel to the edges and is thus stabilized by the magnetostatic energy. However, as shown in Fig. 3, end domains appear at the short edges of the particle arranged in the so-called $S$ state, with both end domains pointing in the same direction. ${ }^{4}$ Two small reversed domains are also nucleated at the corners. Whilst the center of the particle is still largely parallel to the applied field, both ends of the particle break into a partially flux-closed structure including a vortex. These two vortices propagate towards the center of the particle. At $H \sim 100 \mathrm{Oe}$, the nucleation of two other vortices is associated with a strong decrease of the total magnetization. Once this low-field fluxclosed structure is nucleated, it evolves in a reversible way when the field is reversed, as four reversed domains extend from both sides of the particle toward its center. They collapse for $H \sim-350 \mathrm{Oe}$, when nearly negative saturation is achieved.

It is interesting to compare the magnetization processes for the two field orientations. In the longitudinal case, the magnetization reversal nucleates at the edges of the element and propagates toward the center, while in the transverse case, the reversal starts at the center and extends toward the ends of the particle. This gives rise to two different remanent magnetic domain patterns with different numbers of vortices, but which both exhibit a perfect flux closure and nearly zero net magnetization.

These calculations are in good qualitative agreement with experiment. The magnetization curves exhibit the same features as the MOKE loops shown in Fig. 1. Note that the hysteresis observed experimentally is not perfectly reproduced by the calculation (particularly in the transverse case). This discrepancy is most likely due to a slight misorientation of the applied field in the MOKE experiments and to some shape irregularities within the array. In the transverse case, the zero-field domain structure (Fig. 2) is consistent with the MFM picture shown in Fig. 1. However, in the longitudinal case, the simulated domain structure of the remanent state does not agree with that found experimentally; the experimental domain pattern of Fig. 3 contains three vortices, whereas the calculated structure involves four vortices. This discrepancy can be understood in terms of the metastability of the nucleation process of the reversed domains. As shown in Fig. 3, when a large field is applied along the particle axis, the $S$ state is stabilized. Given this $S$ state nucleation pattern, we obtain the zero-field domain structure shown in Fig. 3. However, under certain conditions, it is also possible to stabilize the $C$ state, with the magnetization along the two short edges antiparallel to each other. This $C$ state was obtained in simulations by applying a moderate longitudinal field $H$ $\sim 600 \mathrm{Oe}$, starting from a random initial state. For the $C$ state pattern, the remanent domain structure exhibits only three vortices, in good agreement with experiment. This domain structure has the lowest energy of $E=1.53 \times 10^{-8} \mathrm{erg}$, compared to $E=1.58 \times 10^{-8} \mathrm{erg}$ for the four-vortex pattern and $E=1.74 \times 10^{-8} \mathrm{erg}$ for the two-vortex pattern obtained after transverse saturation.

There are several possible reasons why the experimental results may not agree with the simulations. Thermal fluctuations, which are not included in the simulations, can allow the system to switch between the $S$ and $C$ states and to follow a lower energy magnetization reversal path. Asymmetric shape defects may also be important. Indeed we have found that modifications of the element's shape near one of its' corners allows the stabilization of the $C$ state and the nucleation of a three-vortex zero-field pattern. The role of corner defects on these nucleation processes was also observed experimentally in similar $2 \mu \mathrm{m}$ wide $\mathrm{Fe}$ particles ${ }^{9}$ and in $\mathrm{NiFe}$ elements. ${ }^{4,5}$

In conclusion, micromagnetic simulations of the magnetization switching processes of micron-sized Fe elements are in good agreement with experiment and can be used to predict the behavior of these and related elements. The magnetic history is found to play a crucial role in the nucleation of a flux-closed domain structure in zero field. At least three different flux-closed states can be stabilized in zero field depending upon the magnetic field history. Thus, it is possible to accurately control the magnetic state of these systems by varying the magnetic history.

This work was supported in part by the Office of Naval Research (Contract No. N00014-96-1-1207).

${ }^{1}$ S. S. P. Parkin, K. P. Roche, M. G. Samant, P. M. Rice, R. B. Beyers, R. E. Scheuerlein, E. J. O'Sullivan, S. L. Brown, J. Bucchigano, D. W. Abraham, Yu Lu, M. Rooks, P. L. Trouilloud, R. A. Wanner, and W. J. Gallagher, J. Appl. Phys. 85, 5828 (1999).

${ }^{2}$ P. Ciureanu and S. Middelhoek, Thin Film Resistive Sensors (Institute of Physics Publishing, Bristol, 1992).

${ }^{3}$ Y. Zheng and J.-G. Zhu, J. Appl. Phys. 81, 5471 (1997).

${ }^{4}$ K. J. Kirk, J. N. Chapman, and C. D. W. Wilkinson, Appl. Phys. Lett. 71, 539 (1997).

${ }^{5}$ T. Schrefl, J. Fidler, K. J. Kirk, and J. N. Chapman, J. Magn. Magn. Mater. 175, 193 (1997).

${ }^{6}$ J. Shi, S. Tehrani, T. Zhu, Y. F. Zheng, and J. G. Zhu, Appl. Phys. Lett. 74, 2525 (1999).

${ }^{7}$ A. D. Kent, U. Rüdiger, J. Yu, S. Zhang, P. M. Levy, and S. S. P. Parkin, IEEE Trans. Magn. 34, 900 (1998).

${ }^{8}$ J. Yu, U. Rüdiger, L. Thomas, S. S. P. Parkin, and A. D. Kent, J. Appl. Phys. 85, 5501 (1999).

${ }^{9}$ J. Yu, U. Rüdiger, A. D. Kent, L. Thomas, and S. S. P. Parkin, Phys. Rev. B 60, 7352 (1999).

${ }^{10}$ See 1lg@dancris.com.

${ }^{11}$ A. Aharony, J. Appl. Phys. 83, 3432 (1998). 